UNDERGRADUATE RESEARCH IN NATURAL AND CLINICAL SCIENCE AND TECHNOLOGY (URNCST) JOURNAL Read more URNCST Journal articles and submit your own today at: https://www.urncst.com

\title{
Cognitive Training and Aerobic Exercise as Intervention Techniques for Mild Cognitive Impairment: A Research Protocol
}

\author{
Harneet Cheema, BHSc Student [1]* \\ [1] Department of Health Science, University of Ottawa, Ottawa, ON, K1N 6N5 \\ *Corresponding Author: harneet_cheema@hotmail.com
}

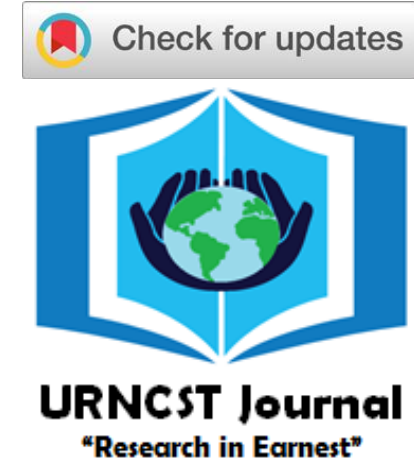

\begin{abstract}
Introduction: Mild cognitive impairment (MCI) is characterized by cognitive decline, prodromal to dementia. However, no medications currently exist. However, research suggests intervention techniques like exercise and cognitive training to slow MCI-progression. The purpose of this research protocol is to determine whether these intervention techniques work more efficiently in combination or separately.

Methods: 80 participants with MCI will be recruited and divided into four groups of 20 participants each; Group-1 will be exposed to cognitive training, Group-2 will be exposed to aerobic exercise, Group-3 will be exposed to both, and Group-4 will be exposed to none. All participants will write a series of cognitive tests that establish a baseline cognition level. After sixmonths of training, participants will rewrite the tests. An analysis of variance will be done on pre- and post-test scores to identify the strategy that produces the most positive change.

Results: Since past literature has found that cognitive training and physical exercise effectively slow cognitive decline, it can be anticipated that a combination of both will be more effective than either intervention alone. It can also be anticipated that all groups involving cognitive training and physical exercise, either alone or in combination, will experience more positive change on their post-test scores than the controls.

Discussion: Literature suggests that a combination of two effective interventions may be more effective than either alone; a study examining the impact of two interventions on falls and cognition in individuals with MCI found both interventions together was the most effective treatment. By conducting a longitudinal study involving a Control-group and multiple cognition-screening tests, this protocol enables the investigation of another possible treatment avenue for individuals with MCI. Conclusion: By examining the interaction between two effective treatment methods for MCI, a condition without medications, this study provides individuals with MCI an additional treatment route that may slow cognitive decline. To permit generalization, future studies should be conducted using larger participant pools that are matched for demographic factors.
\end{abstract}

Keywords: mild cognitive impairment; cognitive training; aerobic exercise; dementia; mixed combination

\section{Introduction}

Mild cognitive impairment (MCI) is commonly described as the prodromal stage to dementia, including Alzheimer's disease (AD), and is characterized by a decline in cognitive functioning beyond expected for an individual's age [1]. Since MCI can be reversible, it is paramount to treat this condition to prevent further cognitive decline in these individuals [1]. Since no effective medications currently exist for MCI [1], it is imperative to investigate alternatives.

According to current research, intervention techniques such as cognitive training [2] and physical exercise [3] can slow the progression of MCI to variable degrees. A clinical trial by Lautenschlager et al. found that exercise has direct positive effects on human memory [4]. Research conducted with animal models has also demonstrated cognitionenhancing effects attributed to exercise; these effects include improved neuron survivability, vascularization, neuroinflammation, and neuroendocrine responses.
Furthermore, animal studies have found that exercising also has positive effects on human physiological processes; if these processes are compromised, the risk of cognitive impairment also increases. Prospective [5] and crosssectional [6] brain imaging studies have discovered positive effects of aerobic exercise in cognitively healthy older adults, particularly in reducing age atrophy, and the maintenance of memory processes and executive control. Studies examining cognitive decline in individuals with AD have found that physical exercise decreases whole-brain atrophy. [7]. The Canadian Study of Health and Aging revealed that physical activity is associated with decreased risk of cognitive impairment and decline in individuals with $\mathrm{AD}$ [8]. Exercise is associated with positive effects in executive control processes including inhibition, working memory, organization, selective attention, and planning [9].

As for cognitive training interventions, research by Brum et al. reports that these interventions significantly 
UNDERGRADUATE RESEARCH IN NATURAL AND CLINICAL SCIENCE AND TECHNOLOGY (URNCST) JOURNAL Read more URNCST Journal articles and submit your own today at: https://www.urncst.com

improve measures of cognitive and emotional processing, as well as functional independence in individuals with MCI. In this study, a cognitive training group underwent eight training sessions within the timespan of one month, with each session lasting two hours. Every session included executive functioning tasks such as memory exercises with visual aids, verbal association tasks, orientation in space and time, exercises for auditory and visual attention, and a transfer task that simulated real-life scenarios. Improvements were noticed in areas of time orientation, attention, dealing with finances, and shopping skills, compared to controls. Furthermore, researchers found a decrease in depressive symptoms across participants [10]. A review by Belleville revealed that, out of the general older adult population, cognitive training yielded the most positive results in participants that were more cognitively preserved. The authors thus concluded that it is within this demographic that cognitive training has the most potential to slow cognitive decline in individuals with MCI [2]. Moreover, another review by Miotto et al. has shown that individuals with MCI retain a degree of neuroplasticity, indicating that cognitive training can be a feasible treatment avenue for this population [11]. Overall, a plethora of evidence supports that cognitive training has positive effects on individuals with MCI by slowing cognitive decline and reducing the rate of progression to dementia.

Many studies have assessed the independent effect of cognitive training or physical exercise on individuals with MCI. However, the interaction between both intervention techniques must be further examined in the literature; the combination of these interventions may be more effective than either one alone. Therefore, the objective of this paper is to propose a research protocol examining how the effects of physical exercise combined with cognitive training may be more effective in alleviating cognitive decline in individuals with MCI compared to either intervention type alone. This research protocol will bridge the gap by examining both techniques separately and in conjunction with each other to determine the most effective treatment route for individuals with $\mathrm{MCI}$.

\section{Methods}

Participants

This study will recruit 80 participants previously diagnosed with MCI via referral sampling, where physicians will recruit participants that fit the inclusion criteria of MCI. The inclusion criteria of MCI will be defined by Petersen's criteria: (1) Objective memory impairment, (2) Memory concerns reported by self or reliable third-party informant (3) Normal cognitive functioning in domains other than memory, (4) Absence of other apparent cognitive disorders, and (5) Absence of dementia [12].

Procedure

During recruitment, participants will notify researchers of any concurrent pharmacological therapies. If participants begin medications between test sessions, they will also notify the researchers immediately to account for this variable. Participants will undergo a six-month intervention for five days a week; this protocol has been chosen to be be able to follow changes overtime in the population. The inclusion criteria will be that all participants do not regularly engage in aerobic exercise or cognitive training. After the recruitment phase is completed, all participants will complete a series of cognitive tests to establish a baseline level of cognition to be compared to post-experiment test scores. One test detects or confirms MCI diagnosis, and all subsequent tests are designed to assess a different aspect of cognitive functioning, including memory, visual recognition, cognitive interference, and spatial functioning. Once the pre-experiment tests are completed, all participants will be randomly divided into four groups, with a target of 20 individuals in each group; participants will be blinded. Group 1, the Physical Exercise group, will be exposed to aerobic physical exercise interventions. The physical exercises will all consist of aerobic exercises due the study by Laura et al., which showed aerobic exercises to have particularly favourable effects on people with MCI [9]. Participants will spend 30 minutes engaging in these exercises daily. Group 2, the Cognitive Training group, will be exposed to cognitive training interventions. These simulate cognitive activities that occur in daily life, as discussed in Brum et al [10]. All participants will complete one cognitive training task each weekday for a total of 30 minutes daily. Group 3, the Mixed Intervention group, will contain participants exposed to both intervention strategies. They will spend 30 minutes engaging in physical exercise and 30 minutes engaging in cognitive training, totalling one hour of participation daily. Group 4, the Control group, will contain the controls that are not exposed to any intervention and will be asked to refrain from engaging in aerobic exercises or cognitive training exercises beyond their day-to-day routine. The researchers will consistently follow up with the participants for six months to ensure the regimens are maintained. After six months, the participants will re-take the same tests from the beginning of the study. Results prior to and after participation will be analysed to identify changes in cognition attributed to the intervention.

\section{Neuropsychological Assessments}

All participants will take both pre and post experiment cognitive screening tests. To begin, the participants will take the Montreal Cognitive Assessment (MoCA), a test designed to detect MCI [13]. The participants will also take the Hopkins Verbal Learning Test (HVLT), a memory impairment screening test that employs memory recall consisting of 12 words, 4 words each from 3 different categories [14]. A visual recognition memory test, the (Delayed Match-to-Sample) DMS 48, will also be employed [15]. Next, participants will complete the Stroop Colour and Word test, which assesses an individual's capability to inhibit the cognitive interference when the processing of one stimulus prevents the processing of another [16]. After this, 
the participants will do the clock-drawing test, used to measure visuospatial dysfunction as a sign of cognitive impairment [17].

\section{Physical Exercise Intervention}

Since the physical capabilities of each participant may vary, it is important to choose a regimen that is feasible for all participants for an extended period. As such, all participants will undergo a physical examination to determine their physical limits to prevent risking their health. As previously mentioned, participants in the Physical Exercise group will exclusively engage in aerobic exercise interventions. The Physical Exercise group and the Mixed Interventions group will engage in 30 minutes of brisk walking daily. These sessions will occur exclusively on weekdays, from Monday to Friday, to allow for a rest period on the weekends. The participants may choose to complete their sessions indoors using machinery such as ellipticals and treadmills. The participants who are comfortable with greater aerobic exercise levels will have the choice of engaging in more intensive exercises, such as jogging or running in lieu of brisk walking. All participants will electronically log which aerobic exercise they completed each day to account for the intensity of the exercises on any changes in test scores.

\section{Cognitive Training Intervention}

Participants from the Cognitive Training group and the Mixed Intervention group will undergo daily cognitive training exercises for six months. Five tasks will be included in the intervention. To test simple verbal associations, the names of the participants and the researcher will be probed. To test general orientation, participants will be asked to state the current year, month, day, and time using external aids. Additionally, memory exercises will be conducted. Auditory and visual attention tasks will also be completed, where participants identify particular details in visual stimuli, find the differences between two similar stimuli, pick up on specific words in songs, and differentiate between different and repeated words. Moreover, transfer tasks will be administered to this group as well, where daily activities such as buying groceries and counting change will be simulated [18]. For each weekday, these participants will complete one of aforementioned cognitive training tasks for 30 minutes, with the tasks counterbalanced and randomized across each of the five weekdays. Much like the Physical Exercise group, weekends will be rest periods.

\section{Data Analysis}

Pre- and post-test scores for each participant will be subjected to data analysis. A two-way analysis of variance (ANOVA) will be employed with Group (Physical Exercise, Cognitive Training, Mixed, Control) as the between-subjects factor and Time (Pre, Post) as the within-subjects factor. If the ANOVA test reaches significance, then post-hoc analyses will be conducted. To determine if the changes found between the pre- and post-test scores are significant for each group, post-hoc tests between Pre- and Post-testing session will be conducted for each group. An additional posthoc one-way ANOVA test with Group as the betweensubjects factor will be conducted to compare the differences between each time point (i.e., difference scores) between the groups to understand which group experienced the most positive change over time.

\section{Results}

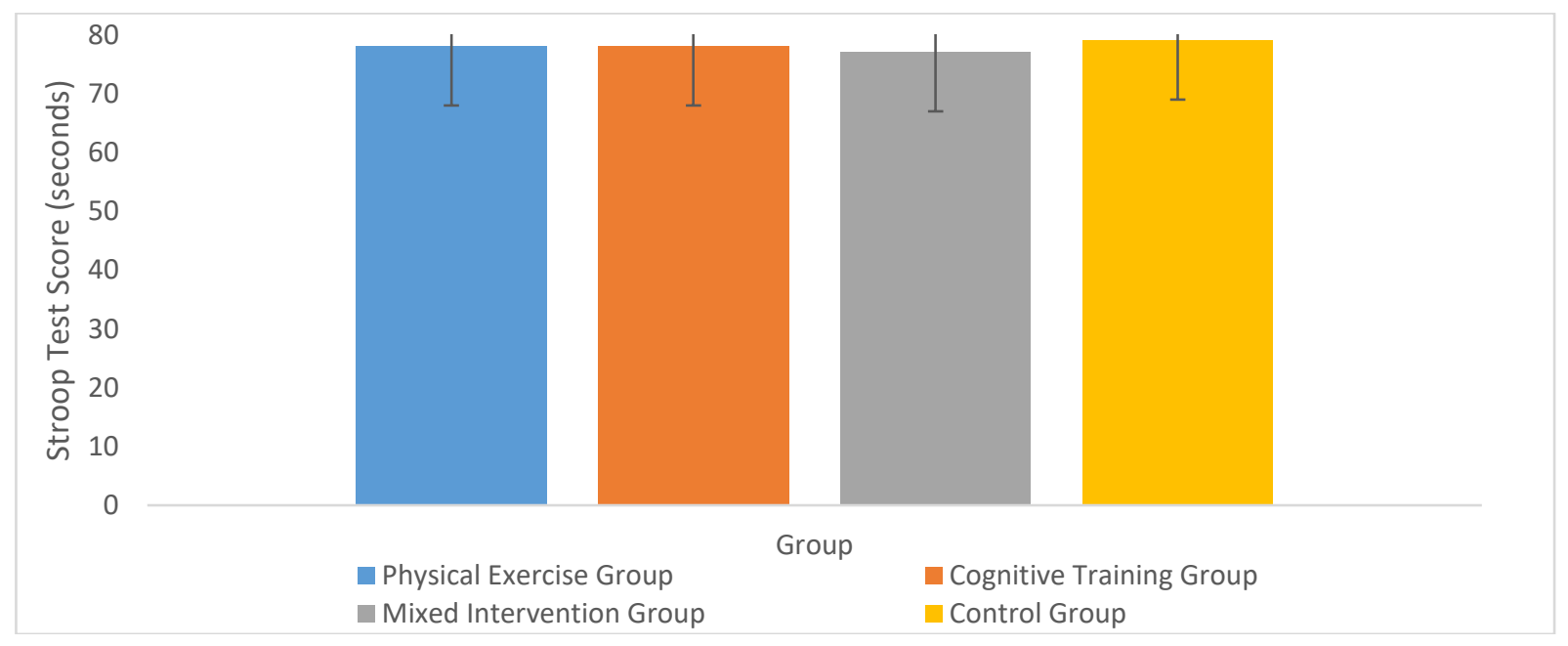

Figure 1. Pre-test anticipated Stroop scores for the physical exercise group, cognitive exercise group, mixed intervention group, and control group 


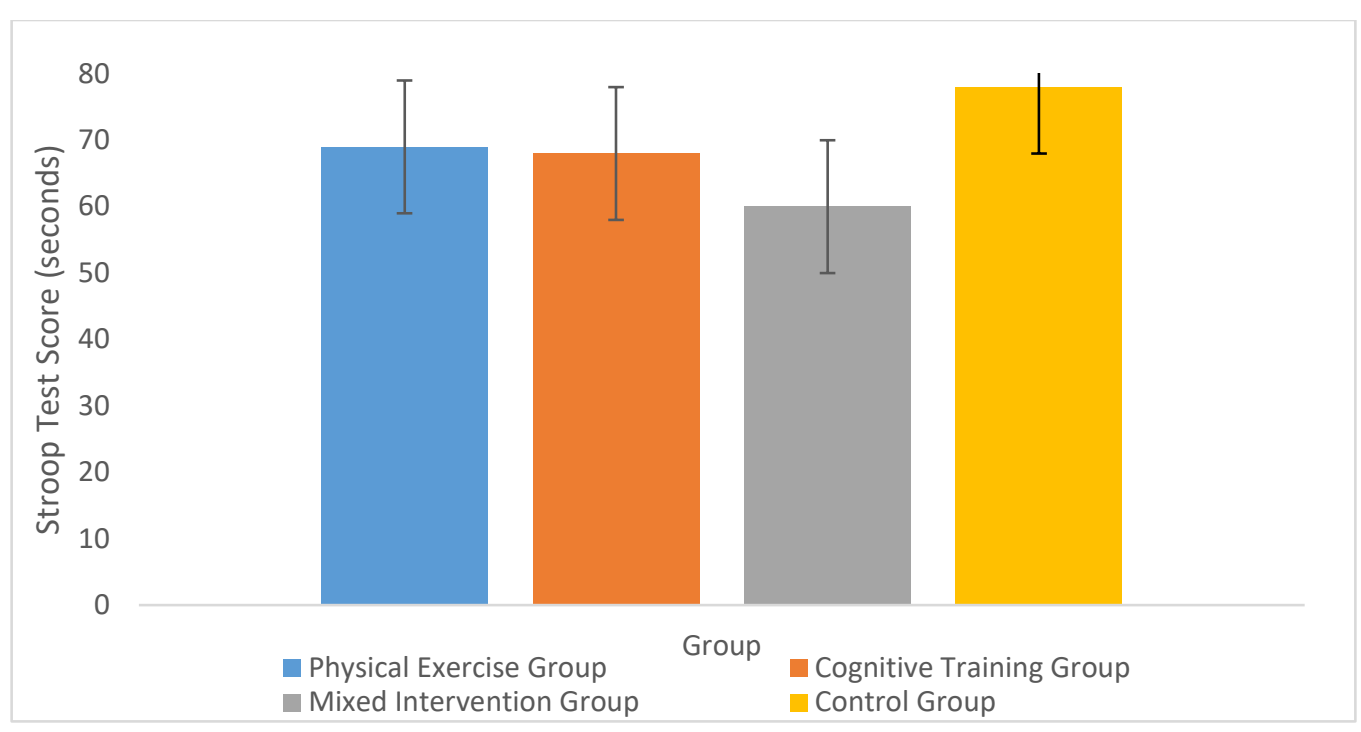

Figure 2. Post-test anticipated Stroop scores for the physical exercise group, cognitive training group, mixed intervention group, and control group

Studies have found that physical exercise alone or cognitive training alone may delay cognitive decline [10]. Therefore, we can anticipate that a treatment strategy combining both interventions would magnify the extent of cognitive decline in participants with MCI. Anticipated results for a sample cognitive test (Stroop test) are displayed in Figure 1 (Pre) and Figure 2 (Post). We anticipate that between the Physical Exercise and Control group, the Physical Exercise group would experience a greater increase in pre- and post-test scores relative to the controls, thereby indicating the potential of Physical Exercise to slow cognitive decline. Between the Cognitive Training and Control group, we can expect the same trend; the participants in the Cognitive Training group will have a larger difference between their pre- and post-test scores compared to the Control group. Considering how past literature suggests that both cognitive training [2] and physical exercise [3] are effective strategies in slowing cognitive decline, we can anticipate that both the Cognitive Training and Physical Exercise group would experience a similar increase between pre- and post-test scores. We do not anticipate for the Control group to experience improvements on cognitive scores over time, since these participants do not undergo any interventions. Therefore, their post-test scores can be expected to be similar to or worse than their pre-test scores. As expected, the Mixed Intervention group will demonstrate improvements in their score over time.

The post-hoc one-way ANOVA on difference scores will reveal whether the change in cognition over time for the Mixed group is beyond either the Cognitive Training group or Physical Exercise group alone. As mentioned previously, the Cognitive Training group and Physical Exercise group may experience similar levels of improvement, while participants in the Mixed Interventions group are expected to experience the highest level of improvement relative to either the Physical Exercise or Cognitive Training group. We anticipate that the results will demonstrate that a combination of two interventions will be more effective than any one intervention on its own. Therefore, the Mixed Interventions group should also experience a higher increase in pre- and post-test scores than the Control group.

\section{Discussion}

MCI may progress to the development of dementia, and between $12-18 \%$ of people over the age of 60 are currently living with MCI [19]. Currently, treatment options for MCI only alleviate symptoms [19]. Effective medications do not exist for MCI, so individuals living with MCI need alternative non-pharmacological treatment methods. Such alternatives can include physical exercise interventions, cognitive training interventions, or potentially a combination of both for maximum treatment efficiency. By examining the interaction between two known treatment avenues for MCI, this research protocol has the potential to improve the efficacy and range of MCI treatments, thereby allowing this population to slow their cognitive decline and possibly delay or prevent the development of all dementias.

It can be predicted that the combination of two known effective treatment strategies may be more effective than either strategy alone, given the literature. Primarily, one systematic review investigated the effect of exercise and cognitive training as a mixed intervention on falls, fall-related factors, and cognition in older adults diagnosed with MCI. Similar to this research protocol, participants were divided into three groups: a cognitive training group, a physical exercise group, and a mixed intervention group. The results of this study indicated that a combined intervention strategy employing cognitive training and physical exercise had the most substantial effect on cognitive functioning. This review also found that the mixed intervention improved fall-related 
UNDERGRADUATE RESEARCH IN NATURAL AND CLINICAL SCIENCE AND TECHNOLOGY (URNCST) JOURNAL Read more URNCST Journal articles and submit your own today at: https://www.urncst.com

factors such as balance; this demonstrates that two interventions may be more effective than one, even in older adults at risk of cognitive decline [20]. Another study also investigated the effectiveness of a combination of aerobic exercise and cognitive training for individuals with MCI. Similar to the review findings, it was found that a combined intervention strategy is the most efficient, showing that this study also found that two interventions may be more effective than one [21]. Both studies are relevant to the current research protocol because they examine physical exercise and cognitive training as intervention techniques for MCI. However, the systematic review does not specifically examine aerobic exercise, which the present research protocol does. The second study, by Hyuntae et al., although examining aerobic exercise, does not employ a control group like this protocol. Furthermore, this protocol uses different neuropsychological assessment tests with lower sensitivities and specificities than the ones employed in the literature. The duration of this study is three months, while the duration of this research protocol is six months. Given that this literature pertains to MCI and discusses similar intervention techniques, it is probable that our present protocol will also find that a mixed intervention technique may be the most efficient in this population compared to either aerobic exercise or cognitive training alone.

This research protocol specifically employed the MoCA test, HVLT test, DMS-48 test, Stroop test and the ClockDrawing test, due to their high sensitivity and specificity for MCI. A study revealed that the MoCA test detects $90 \%$ of all participants with MCI. Furthermore, when using this test on a group of participants with mild $\mathrm{AD}$, the MoCA test demonstrated $100 \%$ sensitivity. In terms of specificity, the MoCA test was at $87 \%$ for MCI [22]. As such, it can be determined that the MoCA test is a cognitive screening tool with both a high specificity and sensitivity. Similar sensitivity and specificity were found for the HVLT; the HVLT demonstrates a sensitivity score of $87 \%$ and a specificity score of $98 \%$ with great discriminability between individuals with normal cognition and individuals with MCI [23]. An additional study also demonstrated that the DMS-48 shows similar levels of sensitivity and specificity [24]. Another test, the Stroop Colour and Word test, was also found to have very similar levels of sensitivity and specificity [25]. The final test, the Clock-Drawing test, demonstrates a $76.5 \%$ sensitivity and $87.1 \%$ specificity for individuals with moderate to severe MCI [26].

A limitation to this study is that only 20 individuals will be exposed to each treatment method; the small sample size hinders the generalization of the findings to the rest of the population. Generally, it is difficult to gather a larger MCI participant pool due to slow recruitment and comorbidities with other neurological/psychiatric disorders. Additionally, although the Control group was not prescribed cognitive training or aerobic exercise, it is possible that these participants engaged in either or both activities at their leisure or in their day-to-day activities at some point during the six-month intervention period, which may impact the results. Moreover, it may be difficult for participants in the Physical Exercise Group to adhere strictly to aerobic exercise. The repetitive nature of the exercises may become mundane for some of the participants; therefore, some may stray from the given routine. Attrition may also be a limitation, considering this research protocol involves a longitudinal design lasting six months. Future studies can address these limitations by conducting the same experiment in larger samples that are matched by demographic factors such as age, sex, and geographical location.

\section{Conclusions}

The aim of this research protocol is to understand whether the currently used MCI intervention techniques demonstrate greater efficacy when used in conjunction with one another rather than separately. As such, the protocol was designed to have four participant groups so that the effects of each intervention technique alone, the combination of both techniques, and no technique at all could be compared. Having a Control Group provided baseline data to which changes in the other groups can be compared. This longitudinal study over six months will allow for more detailed observations of changes in cognition over a longer period of time to be seen. Future studies should continue examining whether the techniques should be used separately or in combination with larger participant pools for longer periods of time. The impact of this research protocol is that the findings could improve the current treatment avenues for MCI, allowing individuals with this disorder more options to slow the prognosis and prevent further cognitive decline. By increasing the amount and breadth of research investigating the interaction between two effective treatment methods for MCI, this study may better equip individuals with MCI to be more proactive in slowing their cognitive decline.

\section{List of Abbreviations Used}

MCI: mild cognitive impairment

AD: Alzheimer's disease

MoCA: Montreal cognitive assessment

HVLT: Hopkins verbal learning test

DMS-48: delayed-match-to-sample-48

ANOVA: analysis of variance

\section{Conflicts of Interest}

The author declares that they have no conflict of interests.

\section{Ethics Approval and/or Participant Consent}

Ethics approval and informed consent will be obtained prior to data collection.

\section{Authors' Contributions}

$\mathrm{HC}$ : made all contributions to the planning and design of the study, the collection of data as well as interpretation and analysis of the data, drafted and critically revised the 
UNDERGRADUATE RESEARCH IN NATURAL AND CLINICAL SCIENCE AND TECHNOLOGY (URNCST) JOURNAL Read more URNCST Journal articles and submit your own today at: https://www.urncst.com

manuscript, and gave final approval of the version to be published.

\section{Acknowledgements}

I would like to thank Ricky Chow (Rotman Research Institute) for providing phenomenal mentorship throughout all stages of writing this manuscript; he contributed very useful knowledge, guidance, advice, support, and resources to me. I would like to thank him for his endless patience and understanding throughout this wonderful journey.

\section{Funding}

This study was not funded.

\section{References}

[1] Staving off dementia when you have mild cognitive impairment. [Internet]. Harvard Health. [cited 2021 Aug 6]. Available from: https://www.health.harvard .edu/staying-healthy/staving-off-dementia-when-youhave-mild-cognitive-impairment

[2] Belleville S. Cognitive training for persons with mild cognitive impairment. 2007 Oct 25. https://doi.org/ 10.1017/s104161020700631x

[3] Baker LD, Frank LL, Foster-Schubert K, Green PS, Wilkinson CW, McTiernan A, Plymate SR, Fishel MA, Watson GS, Cholerton BA, Duncan GE, Mehta PD, Craft S. Effects of aerobic exercise on mild cognitive impairment. Archives of Neurology. 2010;67(1). https://doi.org/10.1001\%2Farchneurol.2009.307

[4] Lautenschlager NT, Cox KL, Flicker L, Foster JK, van Bockxmeer FM, Xiao J, Greenop KR, Almeida OP. Effect of physical activity on cognitive function in older adults at risk for Alzheimer disease. JAMA. 2008;300(9):1027. https://doi.org/10.1001/jama.300.9.1027

[5] Colcombe SJ, Erickson KI, Scalf PE, Kim JS, Prakash R, McAuley E, Elavsky S, Marquez DX, Hu L, Kramer AF. Aerobic exercise training increases brain volume in aging humans. The Journals of Gerontology Series A: Biological Sciences and Medical Sciences. 2006;61(11):1166-70. https://doi.org/10.1093/gerona/61.11.1166

[6] Colcombe SJ, Erickson KI, Raz N, Webb AG, Cohen NJ, McAuley E, Kramer AF. Aerobic fitness reduces brain tissue loss in aging humans. The Journals of Gerontology Series A: Biological Sciences and Medical Sciences. 2003;58(2). https://doi.org/10.1093/gerona/58.2.m176

[7] Laurin D, Verreault R, Lindsay J, MacPherson K, Rockwood K. Physical activity and risk of cognitive impairment and dementia in elderly persons. Archives of Neurology. 2001;58(3). https://doi.org/10.1001/archneur.58.3.498

[8] The Canadian study of health and aging: Risk factors for Alzheimer's disease in Canada. 1994 Nov;44(11):207380. https://doi.org/10.1212/wnl.44.11.2073

Cheema | URNCST Journal (2022): Volume 6, Issue 1 DOI Link: https://doi.org/10.26685/urncst.314
[9] Baker LD, Frank LL, Foster-Schubert K, Green PS, Wilkinson CW, McTiernan A, Plymate SR, Fishel MA, Watson S, Cholerton BA, Duncan GE, Mehta PD, Craft S. Effects of aerobic exercise on mild cognitive impairment. Archives of Neurology. 2010;67(1). https://doi.org/10.1001\%2Farchneurol.2009 $\underline{.307}$

[10] Brum PS, Forlenza OV, Yassuda MS. Cognitive training in older adults with mild cognitive impairment: Impact on cognitive and functional performance [Internet]. Dementia \&amp; neuropsychologia. Associação de Neurologia Cognitiva e do Comportamento; 2009 [cited 2021Dec20]. Available from: https://www.ncbi.nlm.nih.gov/pmc/articles/PMC56192 $\underline{30}$

[11] Miotto EC, Bazán PR, Batista AX, Conforto AB, Figueiredo EG, Martin MDGM, Avolio IB, Amaro E, Tiexeira MJ. Behavioral and neural correlates of cognitive training and transfer effects in stroke patients. Frontiers in Neurology. 2020;11. https://doi.org/10.3389\%2Ffneur.2020.01048

[12] Portet F. Mild cognitive impairment (MCI) in medical practice: A critical review of the concept and new diagnostic procedure. Report of the MCI Working Group of the European Consortium on Alzheimer's Disease. 2006 Jun;77(6):714-718.https://doi.org/ 10.1136\%2Fjnnp.2005.085332

[13] MoCA cognitive assessment [Internet]. MoCA: The Most Sensistive Cognitive Screening Tool. [cited 2021 Aug 6]. Available from: https://www.mocatest.org

[14] Kuslansky G. Detecting dementia with the Hopkins Verbal Learning Test and the mini-mental state examination. Archives of Clinical Neuropsychology. 2004;19(1):89-104.https://doi.org/10.1016/S0887$\underline{6177(02) 00217-2}$

[15] Rullier L, Matharan F, Barbeau EJ, Mokri H, Dartigues J-F, Pérès K, Amieva H. The DMS 48: Norms and diagnostic proprieties for Alzheimer's disease in elderly population from the AMI cohort study. Gériatrie et Psychologie Neuropsychiatrie du Viellissement. 2014;12(3):321-30. https://doi.org/10.1684/pnv.2014.0486

[16] Scarpina F, Tagini S. The Stroop color and word test. Frontiers in Psychology. 2017;8. https://doi.org/10.3389/fpsyg.2017.00557

[17] Konstam V. Lehmann I. Cognitive impairment in heart failure. 2011. https://doi.org/10.1016/B978-1-41605895-3.10060-9

[18] Brum PS, Forlenza OV, Yassuda MS. Cognitive training in older adults with mild cognitive impairment: Impact on cognitive and functional performance. Dementia \& amp; Neuropsychologia. 2009;3(2):124-31. https://doi.org/10.1590\%2FS1980$\underline{57642009 \mathrm{DN} 30200010}$ 
UNDERGRADUATE RESEARCH IN NATURAL AND CLINICAL SCIENCE AND TECHNOLOGY (URNCST) JOURNAL Read more URNCST Journal articles and submit your own today at: https://www.urncst.com

[19] Alzheimer's association [Internet]. Mild cognitive impairment (MCI). [cited 2021 Aug 6]. Available from: https://www.alz.org/alzheimersdementia/what-is-dementia/related conditions/ mild-cognitive-impairment

[20] Lipardo DS, Aseron AM, Kwan MM, Tsang WW. Effect of exercise and cognitive training on falls and fall-related factors in older adults with mild cognitive impairment: A systematic review. Archives of Physical Medicine and Rehabilitation. 2017;98(10):2079-96. https://doi.org/10.1016/j.apmr.2017.04.021

[21] Park H, Park JH, Na HR, Hiroyuki S, Kim GM, Jung MK, Kim WK, Park KW. Combined intervention of physical activity, aerobic exercise, and cognitive exercise intervention to prevent cognitive decline for patients with mild cognitive impairment: A randomized controlled clinical study. Journal of Clinical Medicine. 2019;8(7):940. https://doi.org/10.3390\%2Fjcm8070940

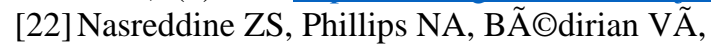
Charbonneau S, Whitehead V, Collin I, Cummings JL, Chertkow H. The Montreal Cognitive Assessment, MOCA: A brief screening tool for mild cognitive impairment. Journal of the American Geriatrics Society. 2005;53(4):695-9. https://doi.org/10.1111/j.1532-5415.2005.53221.x
[23] Shi J, Tian J, Wei M, Miao Y, Wang Y. The utility of the Hopkins Verbal Learning Test (Chinese version) for screening dementia and mild cognitive impairment in a Chinese population. BMC Neurology. 2012;12(1). https://doi.org/10.1186/1471-2377-12-136

[24] Feng X, Zhou A, Liu Z, Li F, Wei C, Zhang G, Jianpin J. Validation of the delayed matching-to-sample task 48 (DMS48) in elderly Chinese. Journal of Alzheimer's Disease. 2018;61(4):1611-8. https://doi.org/10.3233/jad-170530

[25] Rabi R, Vasquez BP, Alain C, Hasher L, Belleville S, Anderson ND. Inhibitory control deficits in individuals with amnestic mild cognitive impairment: A metaanalysis. Neuropsychology Review. 2020;30(1):97125. https://doi.org/10.1007/s11065-020-09428-6

[26] Aprahamian I, Martinelli JE, Neri AL, Yassuda MS. The clock drawing test a review of its accuracy in screening for dementia. Dementia \& amp; Neuropsychologia. 2009;3(2):74-80. https://doi.org/10.1590\%2FS198057642009DN302000 $\underline{02}$

\section{Article Information}

Managing Editor: Jeremy Y. Ng

Peer Reviewers: Ricky Chow, Jala Rizq

Article Dates: Received Aug 07 21; Accepted Nov 13 21; Published Jan 0722

\section{Citation}

Please cite this article as follows:

Cheema H. Cognitive training and aerobic exercise as intervention techniques for mild cognitive impairment: A research protocol. URNCST Journal. 2022 Jan 07: 6(1). https://urncst.com/index.php/urncst/article/view/314

DOI Link: https://doi.org/10.26685/urncst.314

\section{Copyright}

(C) Harneet Cheema. (2022). Published first in the Undergraduate Research in Natural and Clinical Science and Technology (URNCST) Journal. This is an open access article distributed under the terms of the Creative Commons Attribution License (https://creativecommons.org/licenses/by/4.0/), which permits unrestricted use, distribution, and reproduction in any medium, provided the original work, first published in the Undergraduate Research in Natural and Clinical Science and Technology (URNCST) Journal, is properly cited. The complete bibliographic information, a link to the original publication on http://www.urncst.com, as well as this copyright and license information must be included. 
UNDERGRADUATE RESEARCH IN NATURAL AND CLINICAL SCIENCE AND TECHNOLOGY (URNCST) JOURNAL

Read more URNCST Journal articles and submit your own today at: https://www.urncst.com

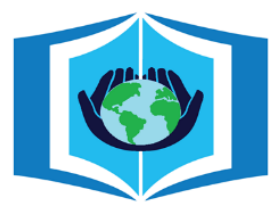

\section{URNCST Journal \\ "Research in Earnest"}

\section{Funded by the Government of Canada}

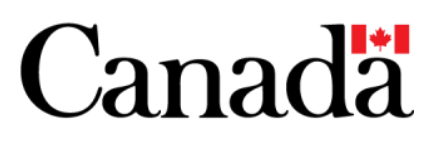

Do you research in earnest? Submit your next undergraduate research article to the URNCST Journal!

| Open Access | Peer-Reviewed | Rapid Turnaround Time | International | | Broad and Multidisciplinary | Indexed | Innovative | Social Media Promoted |

Pre-submission inquiries? Send us an email at info@ urncst.com | Facebook, Twitter and LinkedIn: @URNCST Submit YOUR manuscript today at https://www.urncst.com! 